\title{
Electroweak Physics with Multibosons
}

\author{
Pietro Govoni*广 \\ Università and INFN Milano - Bicocca \\ E-mail: pietro.govoniaunimib.it
}

The CMS Collaboration used the dataset delivered by the Large Hadron Collider in the past years to study rare processes characterised by the simultaneous production of several vector bosons in the proton collisions. This report presents recent results attained in this domain, ranging from inclusive multi-boson final states, to double parton scattering and vector boson scattering.

XXVII International Workshop on Deep-Inelastic Scattering and Related Subjects - DIS2019 8-12 April, 2019

Torino, Italy

* Speaker.

$\dagger^{\dagger}$ on behalf of the CMS Collaboration 


\section{Introduction}

This report presents recent results of the CMS [1] Collaboration in the study of elementary processes charaterised by the simultaneous production of two or more massive vector bosons during the Large Hadron Collider (LHC) operations. While several of such processes are characterised by small production cross sections with respect to the inclusive LHC one, the presence of vector bosons and sometimes specific characteristics of the events allow to define signal regions pure enough to study the electroweak sector of the Standard Model. In the first section of the report the most recent results on inclusive final states are presented, where no additional features besides the presence of vector bosons are sought for. There, the measurement of $\mathrm{Z}$ boson pairs and $\mathrm{W}$ boson triplets production will be described. In the second section double parton scattering production of same-sign $\mathrm{W}$ boson pairs will be summarised, while the third one will be dedicated to vector boson scattering measurements. The comprehensive list of results published by the CMS Collaboration can be found in the dedicated webpages ${ }^{1}$ maintained by the Collaboration.

\section{Inclusive Multi-boson Final States}

Two low cross section processes have been recently studied by the CMS Collaboration, namely the production of $\mathrm{Z}$ boson pairs decaying into electrons or muons [2], and the one of three $\mathrm{W}$ bosons decaying into a system with at least two charged leptons [3]. The first analysis is based on a dataset containing two charged lepton pairs with invariant mass comprised in the range of $(60,120) \mathrm{GeV}$, which is mostly populated by qq-induced and gg-induced signal events, with contaminations due to events with three vector bosons in the final state, or with jets mis-identified as leptons. The mis-identification probabilities are measured in control regions and used to extrapolate the backgrounds from sidebands, yielding a pollution at the order of a few percent. Figure 1 shows on the left-hand side the transverse momentum distribution of single $\mathrm{Z}$ boson candidates in the laboratory rest frame, for the dataset collected during years 2017 and 2018. In the final result, a value for the total production cross section, as well as in a fiducial region, is estimated with the datasets collected in years 2016 (already published [4]), 2017 and 2018. In all cases, the statistical uncertainty is at the same level of the experimental systematic one, dominated by the lepton identification, while the one due to the LHC luminosity measurement is slightly smaller. The uncertainty due to the extrapolation to the total cross section, based on a NLO Powheg generation [5, 6, 7, 8] and LO MCFM one [9] for the qq-induced and gg-induced signal respectively, is comparable to the luminosity one. The overall combination of the results attained in the three years produces a cross section value of $\sigma_{\text {tot }}(p p \rightarrow \mathrm{ZZ})=17.1 \pm 0.3$ (stat) \pm 0.4 (syst) \pm 0.4 (theo) \pm 0.3 (lumi) pb, consistent with the standard model predictions.

The search for the $\mathrm{W}$ boson triplet production features events with three charged leptons identified in the detector, or two same-sign leptons and two jets. To maximise the analysis significance, the signal phase space is split into different regions, where the main contaminations are due to the production of $\mathrm{WZ}$ pairs and the joint production of $t \bar{t}$ pairs with a $\mathrm{Z}$ boson. Figure 1 shows on the right-hand side the observed number of events and predicted yields in the nine signal regions defined by the analysis. While the irreducible background component is relevant in the semi-leptonic

\footnotetext{
${ }^{1}$ https://twiki.cern.ch/twiki/bin/view/CMSPublic/PhysicsResultsSMP
} 

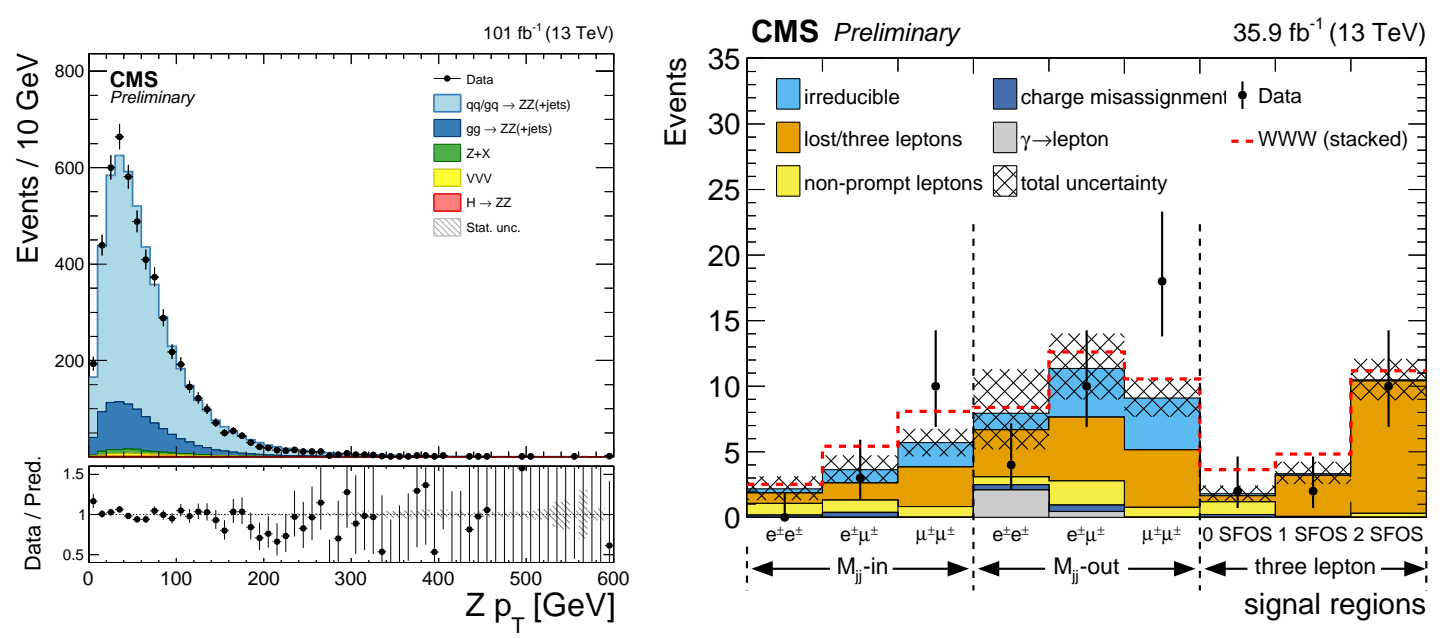

Figure 1: Left: transverse momentum distribution of $\mathrm{Z}$ boson candidates in the CMS search for $\mathrm{Z}$ boson pairs [2]. Events collected during the years 2017 and 2018 populate the distribution of the black points, for a total integrated luminosity of $101.2 \mathrm{fb}^{-1}$. The expected standard model predictions and the background as estimated from data are represented by coloured histograms. Right: observed number of events and predicted yields in the nine signal regions defined by the WWW analysis [3]. The WWW signal shown stacked on top of the total background corresponds to the calculated standard model cross section.

categories with on-shell jet pairs, in the other cases instrumental effects dominate, either because of non-detected charged leptons or mis-identified jets as electrons or muons. These effects are measured from data, while the simulation of the irreducible ones is normalised in control regions, providing a solid estimate of all sources of noise. The best fit to the number of events observed with $35.9 \mathrm{fb}^{-1}$ of integrated luminosity collected in year 2016 yields an underfluctuation with respect to the standard model predictions [10], with a signal strength of $0.34_{-0.34}^{+0.62}$, corresponding to the measured cross section $\sigma_{\mathrm{tot}}\left(p p \rightarrow \mathrm{W}^{ \pm} \mathrm{W}^{ \pm} \mathrm{W}^{\mp}\right)=0.17 \pm 0.32 \mathrm{pb}$. The same events have been used to put limits on beyond-the-standard-model (BSM) effects, parameterised either as singly acting dimension-eight effective field theory (EFT) operators [11], or as a UV-complete model with photophobic axion-like particles [12], with mass large enough that they dominantly decay into $\mathrm{W}$ boson pairs. In both cases, no evidence for an excess of events has been observed and limits have been set for the model parameters.

\section{Double Parton Scattering}

The presence of two same-sign W bosons in a single event during an LHC collision is highly improbable, offering the ideal signature to look for cases where two parton pairs originating from the two colliding protons interact, simultaneously producing a W boson each [13]. The genuine production of such events in a single partonic interaction features additional partons in the final state, and is suppressed in this study by discarding events that contain more than one jet besides exactly two same sign charged leptons (either electrons or muons). The main background left is due to WZ pair production and samples with non-prompt leptons, which are determined from simulation or, in case of important instrumental effects, with data-driven tech- 
niques, and suppressed with multivariate discriminants. After all the selections, the CMS Collaboration determined the first evidence of the double parton scattering WW production with a significance of 3.9 standard deviations with respect to the null hypothesis, analysing $77 \mathrm{fb}^{-1}$ of integrated luminosity collected at $\sqrt{s}$ of $13 \mathrm{TeV}$. This corresponds to an observed cross section of $\sigma_{o b s}($ DPS $)=1.41 \pm 0.28$ (stat) \pm 0.28 (syst) pb.

\section{Vector Boson Scattering}

The scattering of vector bosons (VBS) is one of the rarest processes ever observed at a hadron collider, because of its purely electroweak nature, which implies a small production cross section. Conversely, the final state characterised by the presence of six fermions and the characteristic topology with two energetic jets, the so-called tag jets, well separated in pseudorapidity provide several handles to suppress or evaluate background processes in the data analysis. Depending on the specific vector bosons identified in the final state and their decay mode, different signatures can be investigated, with very different expected number of events, selection purity and overall sensitivity. The selected events are used to measure standard model production cross sections, as well as to set limits on BSM parameters, both in the infrared EFT limit and for UV-complete theories, where hypothetical massive resonances decay into vector boson pairs.

The first VBS signature ever observed was the production of two same-sign $\mathrm{W}$ bosons with two jets, performed by the CMS Collaboration [14]. Despite its rarity, it is as of now the most powerful channel in the standard model study because of the low background and since enough signal events have been collected. As opposed to the double parton scattering case, the presence of two jets besides two charged leptons is required, together with minimal requirements on their pseudorapidity separation (2.5) and invariant mass $(500 \mathrm{GeV})$. The dominant source of contamination in the signal region is due to events with non-prompt leptons originating from leptonic decays of heavy quarks, hadrons misidentified as leptons, and electrons from photon conversions. Its estimate is performed with a data-driven technique [15]. Figure 2 shows on the left the invariant mass distribution of the tag jets in the same-sign WW signal phase space, for the observed events and for the signal and background estimates obtained by the fit of the data. The "others" include QCD WW, W $\gamma$, wrongsign events, double parton scattering, and triboson processes. The signal is clearly visible above the various backgrounds: with $35.9 \mathrm{fb}^{-1}$ of collected events the CMS Collaboration observed this VBS process with a significance of 5.5 standard deviations with respect to the null hypothesis, providing a fiducial cross section measurement of $\sigma_{\text {fid }}(\mathrm{SSWW})=3.83 \pm 0.66$ (stat) \pm 0.35 (syst) fb, which is still statistically dominated. Also in this case, limits on BSM theory parameters are set in the framework of EFT and for UV-complete theories where doubly-charged high-mass resonances may decay into same-sign $\mathrm{W}$ boson pairs [16].

The second channel in terms of sensitivity to the standard model VBS production cross section features WZ pairs decaying into leptons [17], where the dominant background is due to the QCD production of WZ pairs with jets. The CMS Collaboration developed a conservative and robust approach to the event selection, based on well-understood variables to define the signal region. With $35.9 \mathrm{fb}^{-1}$ of integrated luminosity, the electroweak WZ production in association with two jets has been measured with an observed significance of 2.2 standard deviations and and expected one of 2.5. Limits have been set on charged high-mass resonances decaying into WZ pairs and 

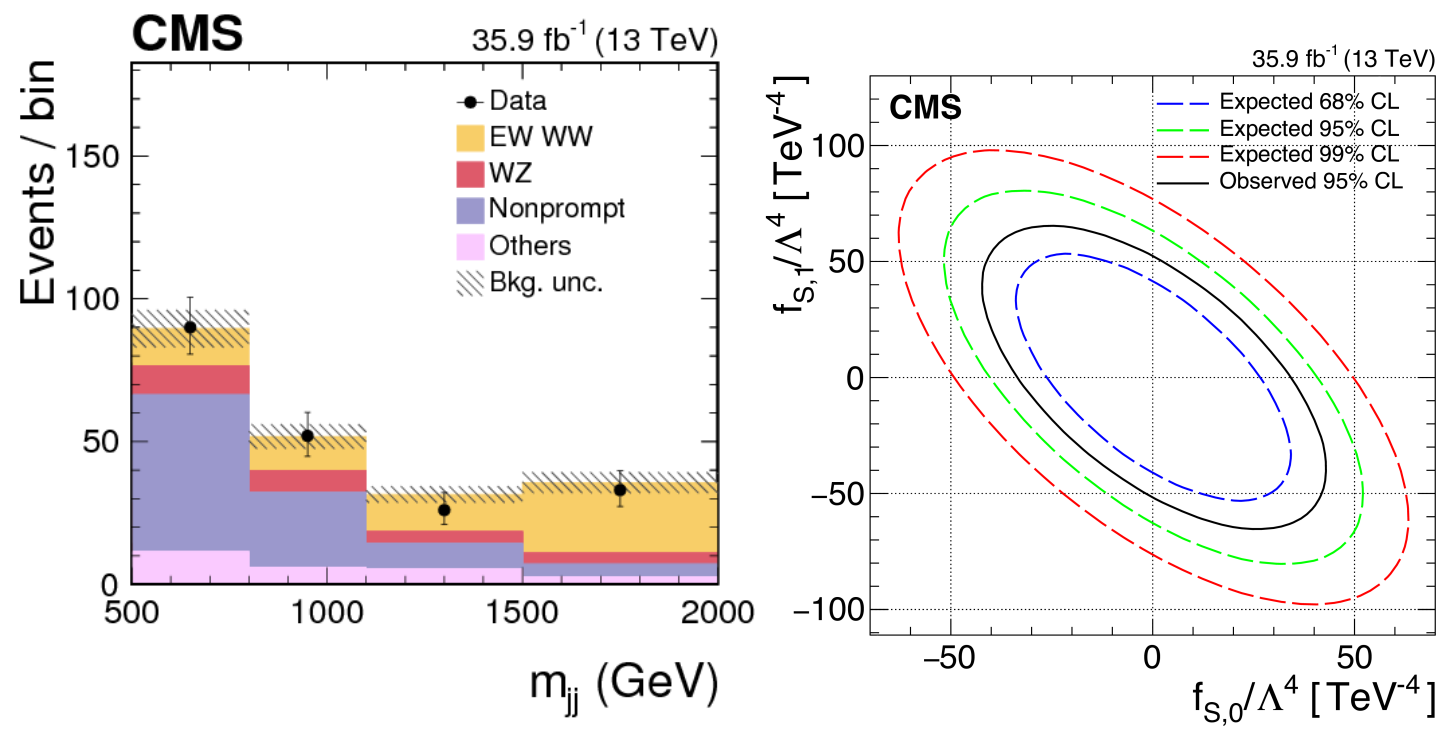

Figure 2: Left: invariant mass distribution of the tag jets in the same-sign WW signal phase space, for the observed events and for the signal and background estimates obtained by the fit of the data [14]. The “others" include QCD WW, WÎş, wrong-sign events, double parton scattering, and triboson processes. The overflow is included in the last bin. Right: two-dimensional 68, 95, and 99\% confidence level boundaries obtained by the study [17], when simultaneously varying two dimension-eight EFT coefficients in the WZ data analysis.

in the EFT framework. Figure 2 shows on the right-hand side two-dimensional 68, 95, and 99\% confidence level boundaries obtained by the study, when simultaneously varying two dimensioneight coefficients.

The rarest process of them all is the one where two $\mathrm{Z}$ bosons decay leptonically in the detector [18], generating a very clear signature of two opposite charge lepton pairs resonating at the $\mathrm{Z}$ mass peak. For how much this topology is pure, is also very improbable, therefore the final data fitting is performed in the analysis on the distribution of a discriminant built with a multivariate approach from several variables characterising the signal. Figure 3 shows, on the right-hand side, the distribution of such variable for the events collected in $35.9 \mathrm{fb}^{-1}$ of integrated luminosity, for the signal, for the irreducible background due to the QCD production of ZZ boson pairs and to events with non-prompt charged leptons. From the fit of the distributions to the data, the signal presence has been determined with a significance of 2.7 standard deviations with respect to the null hypothesis, corresponding to a fiducial cross-section of $\sigma_{\text {fid }}\left(p p \rightarrow \mathrm{ZZjj} \rightarrow \ell \ell \ell^{\prime} \ell^{\prime} j j\right)=$ $0.40_{-0.16}^{+0.21}$ (stat) (syst) ${ }_{-0.09}^{0.13} \mathrm{fb}$.

On the opposite extreme of the spectrum, the final state containing one $\mathrm{W}$ boson decaying leptonically and one vector boson disintegrating into jets [19] shows a substantially larger production cross section, but suffers from a much larger background contamination due to the QCD production of single $\mathrm{W}$ bosons with jets. While this prevented so far any isolation of the standard model VBS process, the large branching ratio makes this channel one very powerful tool to test BSM models, since at large energies, and hence at large $m_{V V}$, the noise pollution, which is measured in a data-driven fashion, is largely reduced. Therefore, stringent limits on BSM model parameters are 

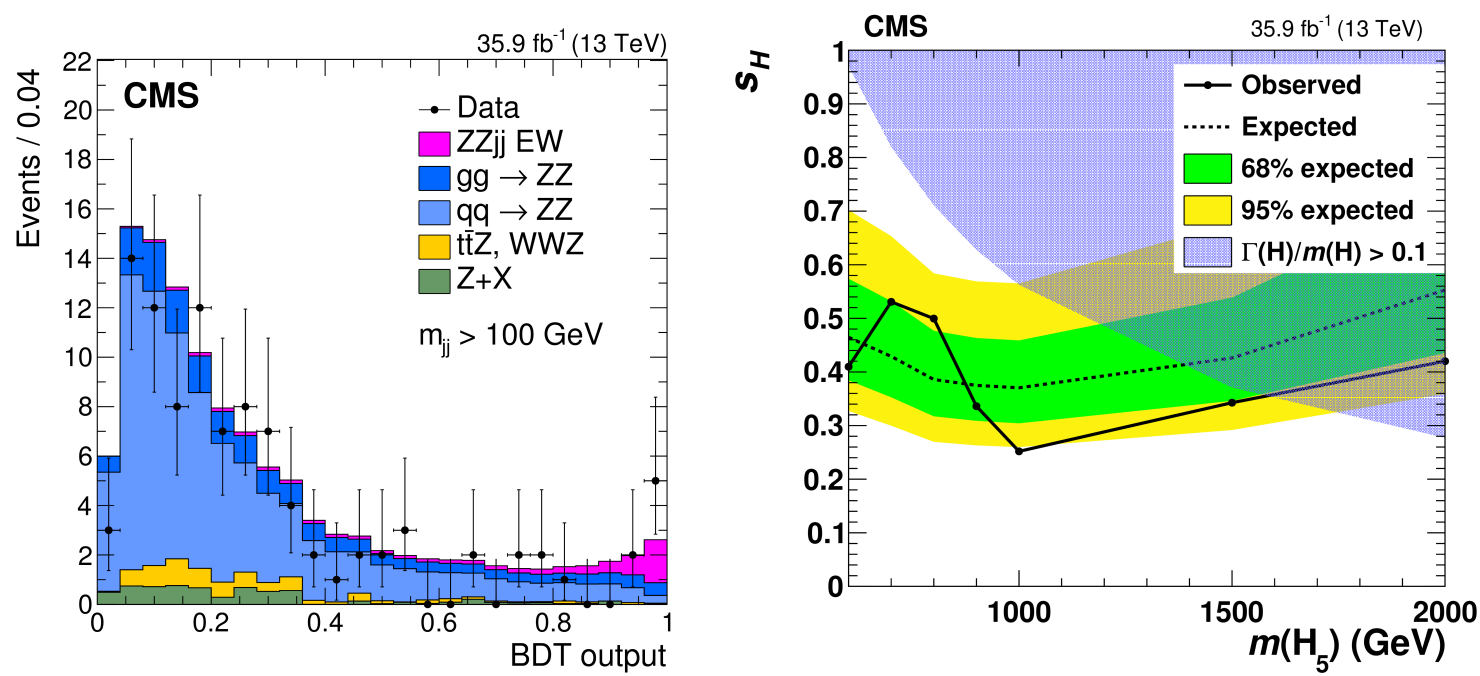

Figure 3: Left: distribution of the multivariate discriminant used for the VBS ZZ study [18]: the counts of the collected events in the various bins is shown, together with the signal, irreducible and non-prompt backgrounds. Right: the expected and observed exclusion limits at the 95\% CL [19] for the $s_{H}$ parameter in the Georgi-Machacek charged Higgs model, together with the theoretically disallowed parameter space [20] represented by a blue shaded area.

set both in the EFT framework and for UV-complete models. Figure 3 shows, on the right-hand side, the expected and observed exclusion limits at the 95\% CL for the $s_{H}$ parameter in the GeorgiMachacek charged Higgs model, together with the theoretically disallowed parameter space [20] represented by a blue shaded area.

\section{Conclusions}

The increasingly large dataset delivered by the LHC in the past years, which will grow even more in the future, allows for the study of events where multiple vector bosons are produced simultaneously in single proton collisions. The study of these rare events is producing the first results and will be an extremely powerful tool not only as a source of precision tests of the standard model, but also as test bench for BSM theories. The experimental collaborations will meet these objectives only if, besides developing at best each single data analysis, they will be able to fully exploit them in a consistent theoretical framework.

\section{References}

[1] S. Chatrchyan et al. [CMS Collaboration], JINST 3 (2008) S08004. doi:10.1088/1748-0221/3/08/S08004

[2] CMS Collaboration, "Measurement of the $\mathrm{pp} \rightarrow \mathrm{ZZ}$ production cross section at $\sqrt{s}=13 \mathrm{TeV}$ with the Run 2 data set," CMS-PAS-SMP-19-001, https://cds.cern.ch/record/2668717.

[3] A. M. Sirunyan et al. [CMS Collaboration], Phys. Rev. D 100 (2019) no.1, 012004 doi:10.1103/PhysRevD.100.012004 [arXiv:1905.04246 [hep-ex]]. 
[4] A. M. Sirunyan et al. [CMS Collaboration], Eur. Phys. J. C 78 (2018) 165 Erratum: [Eur. Phys. J. C 78 (2018) no.6, 515] doi:10.1140/epjc/s 10052-018-5567-9, 10.1140/epjc/s10052-018-5769-1 [arXiv:1709.08601 [hep-ex]].

[5] S. Alioli, P. Nason, C. Oleari and E. Re, JHEP 0807 (2008) 060 doi:10.1088/1126-6708/2008/07/060 [arXiv:0805.4802 [hep-ph]].

[6] P. Nason, JHEP 0411 (2004) 040 doi:10.1088/1126-6708/2004/11/040 [hep-ph/0409146].

[7] S. Frixione, P. Nason and C. Oleari, JHEP 0711 (2007) 070 doi:10.1088/1126-6708/2007/11/070 [arXiv:0709.2092 [hep-ph]].

[8] S. Alioli, P. Nason, C. Oleari and E. Re, JHEP 1006 (2010) 043 doi:10.1007/JHEP06(2010)043 [arXiv:1002.2581 [hep-ph]].

[9] J. M. Campbell and R. K. Ellis, Nucl. Phys. Proc. Suppl. 205-206 (2010) 10 doi:10.1016/j.nuclphysbps.2010.08.011 [arXiv:1007.3492 [hep-ph]].

[10] M. SchÃûnherr, JHEP 1807 (2018) 076 doi:10.1007/JHEP07(2018)076 [arXiv:1806.00307 [hep-ph]].

[11] O. J. P. Eboli, M. C. Gonzalez-Garcia and J. K. Mizukoshi, Phys. Rev. D 74 (2006) 073005 doi:10.1103/PhysRevD.74.073005 [hep-ph/0606118].

[12] N. Craig, A. Hook and S. Kasko, JHEP 1809 (2018) 028 doi:10.1007/JHEP09(2018)028 [arXiv:1805.06538 [hep-ph]].

[13] CMS Collaboration, CMS-PAS-SMP-18-015, https://cds.cern.ch/record/2668320.

[14] A. M. Sirunyan et al. [CMS Collaboration], Phys. Rev. Lett. 120 (2018) no.8, 081801 doi:10.1103/PhysRevLett.120.081801 [arXiv:1709.05822 [hep-ex]].

[15] V. Khachatryan et al. [CMS Collaboration], Phys. Rev. Lett. 114 (2015) no.5, 051801 doi:10.1103/PhysRevLett.114.051801 [arXiv:1410.6315 [hep-ex]].

[16] H. Georgi and M. Machacek, Nucl. Phys. B 262 (1985) 463. doi:10.1016/0550-3213(85)90325-6

[17] A. M. Sirunyan et al. [CMS Collaboration], Phys. Lett. B 795 (2019) 281 doi:10.1016/j.physletb.2019.05.042 [arXiv:1901.04060 [hep-ex]].

[18] A. M. Sirunyan et al. [CMS Collaboration], Phys. Lett. B 774 (2017) 682 doi:10.1016/j.physletb.2017.10.020 [arXiv:1708.02812 [hep-ex]].

[19] A. M. Sirunyan et al. [CMS Collaboration], arXiv:1905.07445 [hep-ex].

[20] M. Zaro and H. Logan, LHCHXSWG-2015-001. 